\title{
Substitution of Acetylene Black by Using Modified Flake Graphite Applied in Activated Carbon Supercapacitors
}

\author{
Peng Zhao ${ }^{1}$, Chunmei $\mathrm{He}^{2}$, and Wencheng $\mathrm{Hu}^{1}$ \\ ${ }^{1}$ State Key Laboratory of Electronic Thin Films and Integrated Devices, University of Electronic Science \& Technology of China, Sichuan, \\ Chengdu, 610054, P. R. China \\ ${ }^{2}$ Experimental School of Shuangliu Middle School, Sichuan, Chengdu, P. R. China
}

\begin{abstract}
Flake graphite was mechanically modified at different times in N-methyl pyrrolidone under normal pressure. The results of the scanning electron microscopy, X-ray diffraction, and transmission electron microscopy suggested that the structure of the flake graphite was modified. The crystallinity of the flake graphite, and many defects were introduced into the material. The evaluation of capacitor performance by cyclic voltammetry, galvanostatic charge/discharge tests, and electrochemical impedance spectroscopy was also performed. Results showed that the electrochemical performance of flake graphite was strongly enhanced, particularly when it was exfoliated for $6 \mathrm{~h}$. Moreover, the electrochemical capacitive properties of activated carbon were obviously enhanced through the substitution of acetylene black by flake graphite modified for $6 \mathrm{~h}$.
\end{abstract}

\section{Introduction}

Supercapacitors (SCs), also known as electrochemical capacitors (ECs), hybrid capacitors, or ultracapacitors, are highly promising power devices that are superior to conventional physical capacitors and batteries in many aspects. These devices also play an important role in the energy-storage field $[1,2]$. SCs show high power density, fast charging/discharging rate, sustainable cycling life, and excellent cycle stability [3, 4]. According to energy storage mechanism, SCs can be divided into two different types: double-layer capacitors (EDLCs) and faraday pseudocapacitor. EDLCs exhibit the capacitance that comes from pure electrostatic charges accumulated at the interface between an electrolyte and an electrode; while the pseudocapacitors typically show higher capacitances owing to the fast and reversible faradic redox reactions of electro-active materials of the electrodes [5-7].

Carbon-based electrode materials are most frequently used materrials for EDLCs include activated carbon, modified porous carbon, carbon nanotubes (CNTs), graphene, carbon aerogels, carbon nanofibers, etc[8]. Consequently, carbon materials are the ideal candidate electrode materials in SCs because of their easy accessibility, good processing ability, large surface area/porosity, low electrical resistivity, robust surface chemical environment, physicochemical stability, versatile existing forms, and low cost $[9,10]$. Graphite is a carbon material that is widely used as the electrode material of SCs [11]. Graphite has a larger surface area and higher specific capacitance for an edge plane than other carbonaceous materials [12].

With regard to ESs, considering that the capacitance intensively relies on the electrolyte accessible surface of an electrode material, many researchers have focused on increasing the total surface area of graphite. For instance, mechanical grinding has been demonstrated as an effective approach [13-16]. After modification, the first cycle efficiency and capacity are improved. Nevertheless, some limitations exist in these methods [12, 17-20]. Thus, better methods are needed to overcome these defects.

We introduce a novel and simple method of modifying graphite by using a high-shear dispersing homogenizing emulsification machine for laboratory. The obtained graphite with high surface area has high specific capacitance and good Faradaic interfacial charge-transfer resistance (Rct). Moreover, this material can improve the electrochemical performance of activated carbon (AC) while doped into $\mathrm{AC}$ at an appropriate ratio. In this paper, flake graphite was chosen as the raw material in this dissertation. The High Shear Disperse Homogenizing Emulsification Machine for Laboratory was used to exfoliate it with different times. According to the results, its crystallinity was reduced, the specific surface area was increased and electrochemical performance was enhanced after flake graphite was exfoliated. Particularly, flake graphite exfoliated six hours (S6) owns the best performance as the specific capacitance 
of S6 is $99 \mathrm{~F} \mathrm{~g}^{-1}$, which is double of the raw material. Then S6 and acetylene black were added to the activated carbon using as conductive agent respectively. The result indicated that the electrochemical performance with S6was better, and the best content is $20 \%$.

\section{Experimental section}

\subsection{Sample preparation}

All analytical-grade chemicals were supplied by Chengdu Changzheng Chemical Industrial, Ltd. (China), and used as received without further purification. Capacitor-grade AC with a specific surface area of approximately $2000 \mathrm{~m}^{2} \mathrm{~g}^{-1}$ was purchased from Carbosino Material Co., Ltd. (China).

In a typical procedure, $0.5 \mathrm{~g}$ of flake graphite was dispersed in an appropriate volume of NMP, and the dispersion was heated in an oil bath at $60{ }^{\circ} \mathrm{C}$ overnight under intensive stirring. Thereafter, the dispersion was exfoliated with a high-shear-disperse homogenizing emulsification machine for laboratory for different times $(2,4,6,8$, and $15 \mathrm{~h})$ in a water bath at $70^{\circ} \mathrm{C}$ under stirring. Thereafter, the resulting dispersion was centrifuged, repeatedly washed with ethanol, and dried in a vacuum at $80{ }^{\circ} \mathrm{C}$. The obtained flake graphite modified for 2, 4, 6, 8, and $15 \mathrm{~h}$ were designated as $\mathrm{S} 2$, S4, S6, S8, and S15, respectively. The untreated flake graphite was referred to as $\mathrm{S} 0$.

\subsection{Characterization}

The X-ray diffraction (XRD) patterns of the five samples were obtained by using a Philips X'pert X-ray diffractometer operated at $1.5406 \mathrm{~nm}, 30 \mathrm{kV}$, and 20 $\mathrm{mA}$. Data were collected at $2 \theta=10^{\circ}$ to $90^{\circ}$. A field-emission scanning electron microscopy system (Inspect F, FEI Co., USA) with an acceleration voltage of $20 \mathrm{kV}$ was used to characterize the surface morphologies. Flake graphite exfoliated in NMP under normal pressure for $15 \mathrm{~h}$ was examined by transmission electron microscopy (TEM; JEM-100CX, JEOL, Japan).

\subsection{Preparation of electrodes}

At the first stage, the working electrodes were prepared by mixing $95 \mathrm{wt}-\%$ modified flake graphite with $5 \mathrm{wt}-\%$ SBR. Thereafter, the working electrodes were prepared by mixing 85 wt- $\%$ AC, 10 wt- $\%$ acetylene black (10 wt $\%, 20 \mathrm{wt}-\%$, and $30 \mathrm{wt}-\% \mathrm{~S} 6)$, and $5 \mathrm{wt}-\%$ SBR and were denoted as a, b, c, and d, respectively. On the basis of the weight of the electrode before and after coating, the mass of the deposited material can was calculated.

\subsection{Electrochemical measurements}

Electrochemical experiments were conducted by using Metrohm Autolab (PGSTAT 302N) with a classical three-electrode electrochemical cell at room temperature and normal pressure. The electrolytes were $7 \mathrm{M} \mathrm{KOH}$ and $1 \mathrm{M}$ aqueous $\mathrm{Na}_{2} \mathrm{SO}_{4}$ solution at the two stages. A platinum tablet $(1 \mathrm{~cm} \times 2 \mathrm{~cm})$ and a saturated calomel electrode were used as the counter electrode and reference electrode, respectively.

\section{Results and discussion}

XRD can be used to obtain the structural information of a material. Fig. 1 presents the XRD patterns of the five samples. Two diffraction peaks were observed near $25.5^{\circ}$ and $54.6^{\circ}$, which corresponded to (002) and (004) reflections. The intensity of the two Bragg peaks gradually weakened, particularly the (002) Bragg peak, with increasing modification time, thus suggesting that many lattice defects were caused by the modification [19]. These defects led to the lower diffraction intensity of the samples than that of flake graphite.

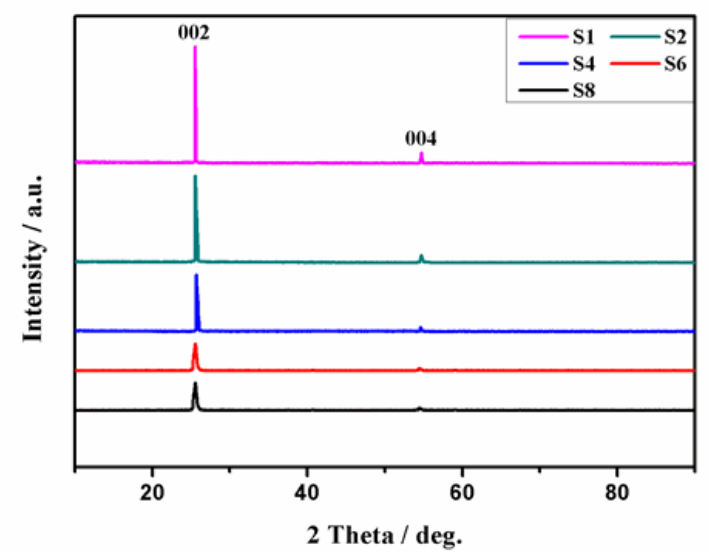

Figure 1. XRD patterns of the original and modified flake graphite.

SEM was used to show the morphologies of the materials (Figs. 2(a-c)). The flake graphite was composed of scaly grains measuring $10 \mu \mathrm{m}$ to $20 \mu \mathrm{m}$. After treating flake graphite, the grain size was reduced and the large flakes were fragmented. With increased modification time, the specific surface area of flake graphite increased. The reason was that the high-shear disperse homogenizing emulsification machine for laboratory can provide high shear forces $(22,000 \mathrm{rpm})$, which undermine the Van der Waals forces of graphite layers. Given the greater transfer channels for ions, the charge/discharge performance at a large current may be strengthened.

Figs. 2(d) show the TEM images of the flake graphite exfoliated in NMP under normal pressure for $15 \mathrm{~h}$. Flake graphite, which has a layer-to-layer film structure, has been exfoliated and cleaved into small pieces of sheet because of the high-energy shear force that breaks the covalent bond within the graphene 
planes [12]. This finding further proves the significant disruption of the ordered graphite structure throughout the long-term modification.

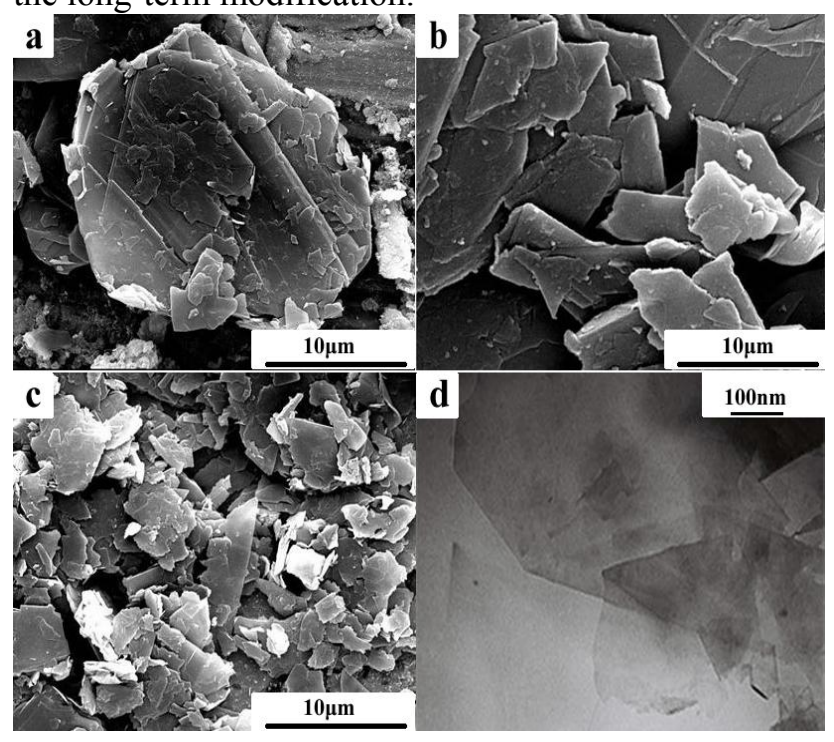

Figure 2. (a) SEM micrograph of S0; (b) SEM micrograph of S2; (c) SEM micrograph of S6; (d) TEM micrographs of S15.

Fig. 3(a) shows the CV curves of S0, S2, S4, S6, and $\mathrm{S} 8$ electrodes at a scan rate of $50 \mathrm{mV} \mathrm{s}^{-1}$ with potential windows ranging from $-1 \mathrm{~V}$ to $0 \mathrm{~V}$ (vs. SCE) in a $7 \mathrm{M}$ aqueous $\mathrm{KOH}$ solution. Flake graphite showed a nearly symmetrical rectangular curve, thus indicating good capacitive behavior. The distortion of the curve shape became progressively serious with increasing modification time. This distortion can be attributed to the deteriorated conductivity of flake graphite because of the intense destruction of the flake graphite structure with prolonged exfoliation time [8]. The area of the curve increased at the previous $6 \mathrm{~h}$ and then decreased because of the variation in the specific capacitance. The specific surface area of the electrode material was not the only factor that influenced the specific capacitance. The variation in the specific capacitance of S8 can be explained by its poorer pore structure than that of S6. Fig. 3(b) depicts the CVs of $\mathrm{S} 6$ at scan rates of $1,5,10,50$, and $100 \mathrm{mV} \mathrm{s}^{-1}$ with potential windows ranging from $-1 \mathrm{~V}$ to $0 \mathrm{~V}$ (vs. SCE) in $7 \mathrm{M} \mathrm{KOH}$ aqueous solution.

The specific capacitance of the electrode measured by $\mathrm{CV}$ can be calculated from following equation:

$$
C=\frac{1}{2 \cdot \Delta V \cdot m} \int_{V_{\text {inital }}}^{V_{\text {final }}} \frac{|I|}{(d V / d t)} d V
$$

where $C$ is the specific capacitance $\left(\mathrm{F} \mathrm{g}^{-1}\right), \Delta V$ is the potential window $(\mathrm{V}), m$ is the mass of the carbon electrode $(\mathrm{g}), V_{\text {initialffinal }}$ is the starting/end potential in one cycle, $|I|$ is the instantaneous current at a given potential, and $d V / d t$ is the potential scan rate. According to the equation, the specific capacitances of $\mathrm{S} 0, \mathrm{~S} 2, \mathrm{~S} 4, \mathrm{~S} 6$, and S8 at a scan rate of $50 \mathrm{mV} \mathrm{s}^{-1}$ were $37,45,52,65$, and $63 \mathrm{~F} \mathrm{~g}^{-1}$.
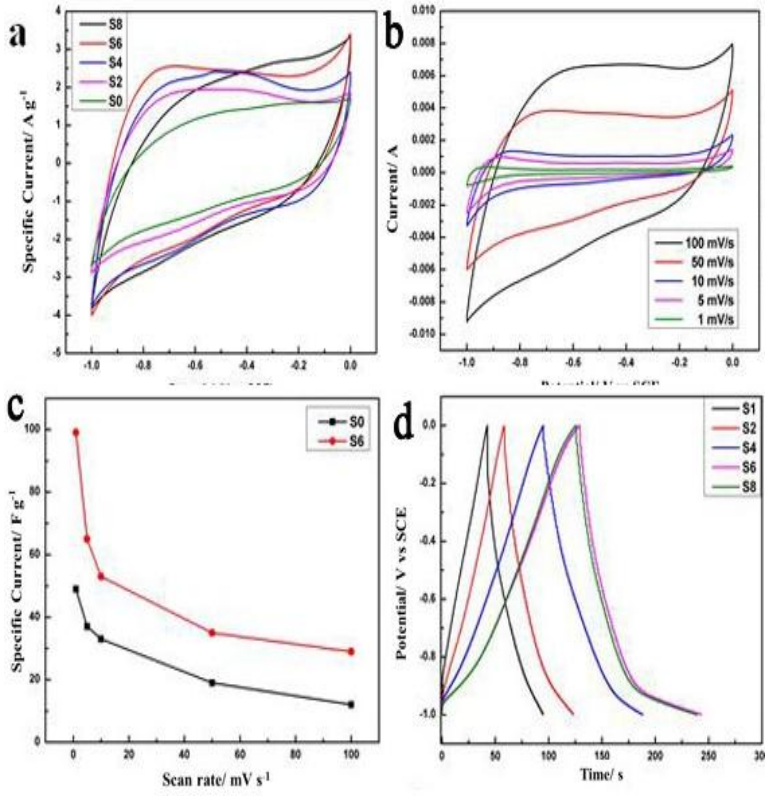

Figure 3. (a) $\mathrm{CV}$ curves of the five electrodes at the scan rate of $50 \mathrm{mV} \mathrm{s}^{-1}$ in $7 \mathrm{M}$ aqueous $\mathrm{KOH}$ solution; (b) $\mathrm{CV}$ curves of the $\mathrm{S} 6$ at different scan rates in $7 \mathrm{M}$ aqueous $\mathrm{KOH}$ solution; (c) The specific capacitance of the S0 and S6 at different scan rates derived from cyclic voltammetry curves; (d) Galvanostatic charge-discharge curves of S0, S2, S4, S6 and S8 electrodes at a current density of $1 \mathrm{~A} \mathrm{~g}^{-1}$.

Fig. 3(c) presents the specific capacitance of S0 and $\mathrm{S} 6$ at different scan rates. At scan rates of 100, 50, 10,5 , and $1 \mathrm{mV} \mathrm{s}^{-1}$, the specific capacitances of S0 were $49,37,33,19$, and $12 \mathrm{~F} \mathrm{~g}^{-1}$, respectively; those of $\mathrm{S} 6$ were $99,65,53,35$, and $29 \mathrm{~F} \mathrm{~g}^{-1}$,respectively. The specific capacitance of S6 was nearly double that of S0. The galvanostatic charge/discharge curves of S0, S2, $\mathrm{S} 4, \mathrm{~S} 6$, and S8 electrodes at a current density of $1 \mathrm{~A} \mathrm{~g}^{-1}$ were obtained within a stable window of $-1 \mathrm{~V}$ to $0 \mathrm{~V}$ (Fig. 3(d)). S6 showed the highest specific capacitance corresponding to the CVs.

Fig. 4(a) shows Nyquist plots for S0, S2, S4, S6, and $\mathrm{S} 8$ electrodes in $7 \mathrm{M} \mathrm{KOH}$ aqueous solution with a frequency loop from $10^{5} \mathrm{~Hz}$ to $10^{-2} \mathrm{~Hz}$. All five Nyquist plots consisted of a semicircle in the high-frequency region and an arc in the low-frequency region. The two parts were ascribed to the charge-transfer process at the electrode/electrolyte interface and the diffusion process in solid, respectively [22]. The semicircle diameter showed the Faradaic interfacial Rct of the electrochemical system. The semicircle diameters of the exfoliated flake graphite electrodes were smaller than those of untreated flake graphite. This finding indicated that electrochemical reaction more easily occurred at the electrode/electrolyte interface of the modified flake graphite. The Rct values of S4, S6, and S8 were very close to one another. The experiment result further indicated that the modified flake graphite possessed excellent electrochemical capacitive properties. 

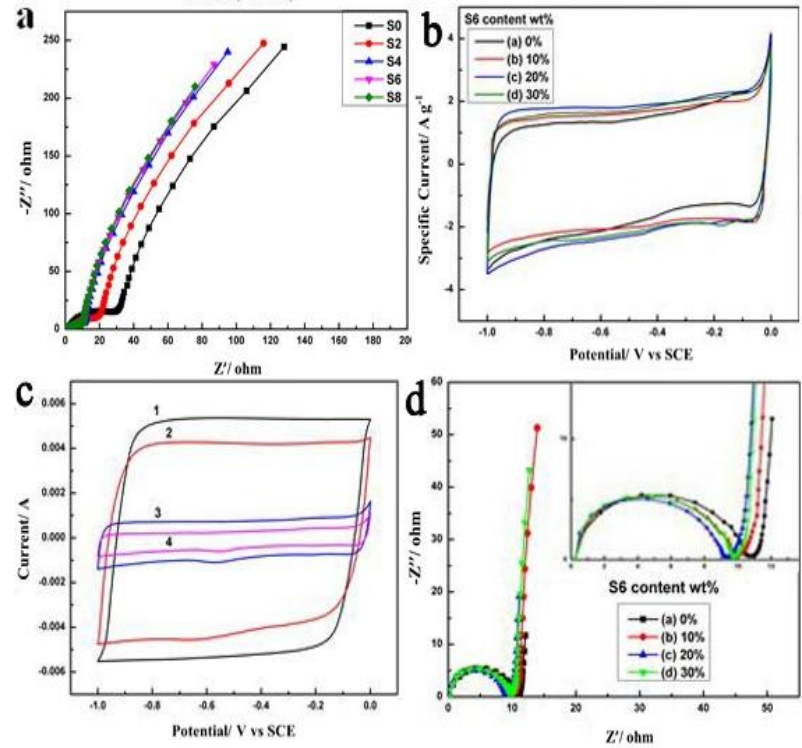

Figure 4. (a) Nyquist plots of S0, S2, S4, S6 and S8 electrodes. (b) $\mathrm{CV}$ curves of S6/AC electrodes with different $\mathrm{S} 6$ content at the scan rate of $10 \mathrm{mV} \mathrm{s}^{-1}$ in $1 \mathrm{M} \mathrm{Na}_{2} \mathrm{SO}_{4}$ aqueous solution; (c) $\mathrm{CV}$ curves of the S6/AC electrode with $20 \mathrm{wt}-\%$ content of S6 at different scan rates in $1 \mathrm{M} \mathrm{Na}_{2} \mathrm{SO}_{4}$ aqueous solution: (1) 100 $\mathrm{mV} \mathrm{s}^{-1}$; (2) $50 \mathrm{mV} \mathrm{s}^{-1}$; (3) $10 \mathrm{mV} \mathrm{s}^{-1}$; (4) $5 \mathrm{mV} \mathrm{s}^{-1}$. (d) Nyquist plots of S6/AC electrodes with different S6 content. The inset shows the high frequency part of the Nyquist plots.

Fig. 4(b) shows the $\mathrm{CV}$ curves of S6/AC electrodes with different S6 contents at a scan rate of $10 \mathrm{mV} \mathrm{s}^{-1}$ with potential windows ranging from $-1 \mathrm{~V}$ to $0 \mathrm{~V}$ (vs. SCE) in $1 \mathrm{M}$ aqueous $\mathrm{Na}_{2} \mathrm{SO}_{4}$ solution. All curves showed a nearly rectangular shape and mirror-image characteristics, suggesting excellent electrochemical behavior. With increased S6 content, the area of the curve increased to different degrees. At a scan rate of $10 \mathrm{mV} \mathrm{s}^{-1}$, the specific capacitances of the four electrodes were 168, 180, 208, and $195 \mathrm{~F} \mathrm{~g}^{-1}$. The specific capacitance of S6/AC electrode with 20 wt-\% S6 content was $23.8 \%$ higher than that of pure AC electrode. The enhancement in specific capacitance can be ascribed to the increase in conductivity and surface area because of the addition of the S6. Fig. 4(c) depicts the CVs of S6/AC electrode with 20 wt- $\%$ content of S6 at scan rates of 5, 10, 50, and $100 \mathrm{mV} \mathrm{s}^{-1}$ with potential windows ranging from $-1 \mathrm{~V}$ to $0 \mathrm{~V}$ (vs. SCE) in $1 \mathrm{M}$ aqueous $\mathrm{Na}_{2} \mathrm{SO}_{4}$ solution.

Fig. 4(d) shows the Nyquist plots of S6/AC electrodes with different $\mathrm{S} 6$ contents in $1 \mathrm{M}$ aqueous $\mathrm{Na}_{2} \mathrm{SO}_{4}$ solution at a frequency loop of $10^{6} \mathrm{~Hz}$ to $10^{-1}$ Hz. All Nyquist plots consisted of a semicircle in the high-frequency region and a line in the low-frequency region. The diameters of semicircles decreased from a to $\mathrm{c}$ and then enlarged from $\mathrm{c}$ to $\mathrm{d}$, thus indicating that the Rct of S6/AC electrode with $20 \mathrm{wt} \%$ S6 content was the smallest one and that $\mathrm{S} 6$ addition can enhance the electrochemical capacitive properties of AC.

\section{Conclusions}

In this study, a simple method was used to modify flake graphite. Flake graphite was exfoliated in NMP by using the high-shear disperse homogenizing emulsification machine for laboratory. The specific capacitance of flake graphite increased from $49 \mathrm{~F} \mathrm{~g}^{-1}$ to $99 \mathrm{~F} \mathrm{~g}^{-1}$ at a scan rate of $1 \mathrm{mV} \mathrm{s}^{-1}$ when it was modified for $6 \mathrm{~h}$. The specific capacitance of S6/AC electrode with 20 wt- $\%$ S6 content was $23.8 \%$ higher than that of pure AC electrode, thus suggesting better electrochemical performance. The enhancement could be ascribed to the intrinsic specific capacitance of the modified flake graphite and the improvement in the hole utilization of AC resulting from the substitution of acetylene black by the modified flake graphite.

\section{References}

1. M. Winter and R. J. Brodd, Chem. Rev. 104, 4245 (2004)

2. T.S. He, W.F. Mu, C.J. Zhang, Z.X. Jin and K.D. Cai, Adv. Mater. Res. 347, 3645 (2012)

3. C. Liu, F. Li, L.P. Ma and H.M. Cheng, Adv. Mater. 22, E28 (2010)

4. K. Liang, X.Z. Tang and W.C. Hu, J. Mater. Chem. 22, 11062 (2012)

5. S. Yoda, K. Ishihara, J. Power Sources 81, 162-169 (1999)

6. J. M. Ko, R. Y. Song, H. J. Yu, J. W. Yoon, B. G. Min and D. W. Kim, Electrochim. Acta 50, 873 (2004)

7. A. Burke, J. Power Sources 91, 37 (2000).

8. H.Q. Li, Y.G. Wang, C.X. Wang and Y.Y. Xia, J. Power Sources 185, 1557 (2008)

9. E. Frackowiak and F. Beguin, Carbon 39, 937 (2001)

10. A.G. Pandolfo and A.F. Hollenkamp, J. Power Sources 157, 11 (2006).

11. H.Y. Wang and M. Yoshio, Electrochem. Commun. 8, $1481(2006)$

12. H.Q. Li, Y.G. Wang, C.X. Wang and Y.Y. Xia, J. Power Sources 185, 1557 (2008)

13. E. Gomibuchi, T. Ichikawa, K. Kimura, S. Isobe, K. Nabeta, and H. Fujii, Carbon 44, 983 (2006)

14. M. Francke, H. Hermann, R. Wenzel, G. Seifert, and K. Wetzig, Carbon 43, 1204 (2005)

15. F. Salver-Disma, A. Du Pasquier, J.M. Tarascon, J.C. Lassègues, and J.N. Rouzaud, J. of power sources, 291 (1999)

16. F. Salver-Disma, J.M. Tarascon, C. Clinard, and J.N. Rouzaud, Carbon 37, 1941 (1999)

17. R. Alcántara, P. Lavela, G. F. Ortiz, J. L. Tirado, R. Menéndez, R. Santamaría, and J. M. Jiménez-Mateos, Carbon 41, 3003 (2003)

18. T.S. Ong and H, Yang, Carbon 38, 2077 (2000)

19. F. Chevallier, L. Aymard, and J. Tarascon, J. Electrochem. Soc. 148, A1216 (2001)

20. Y. J. Kim, Y. Horie, S. Ozaki, Y. Matsuzawa, H. Suezaki, C. Kim, N. Miyashita, and M. Endo, Carbon 42, 1491 (2004)

21. C.S. Wang, G.T. Wu and W.Z. L, J. of Power Sources 76, 1 (1998)

22. A.B. Yuan and Q.L. Zhang, Electrochem. Commun. 8,1173 (2006) 